\title{
Eye-Tracking Analysis to Compute the Visual Hierarchy of User Interfaces on Automated External Defibrillators
}

\author{
Hannah Torney \\ School of Computing \\ Ulster University \\ Newtownabbey, UK \\ torney-h1@ulster.ac.uk \\ Justin Magee \\ Belfast School of Art \\ Ulster University \\ Belfast, UK \\ jdm.magee@ulster.ac.uk
}

\author{
Adam Harvey \\ HeartSine Technologies Ltd. \\ Airport Road West, \\ Belfast, UK \\ adam.harvey@stryker.com \\ Rebecca Funston \\ HeartSine Technologies Ltd. \\ Airport Road West, \\ Belfast, UK \\ rebecca.funston@stryker.com
}

\author{
Dewar Finlay \\ School of Engineering \\ Ulster University \\ Newtownabbey, UK \\ d.finlay@ulster.ac.uk \\ Raymond Bond \\ School of Computing \\ Ulster University \\ Newtownabbey, UK \\ rb.bond@ulster.ac.uk
}

\begin{abstract}
Sudden cardiac arrest (SCA) is a leading cause of death worldwide. It is treated with a defibrillating shock from an automated external defibrillator (AED) and cardiopulmonary resuscitation. AEDs are commonly used by minimally-trained rescuers to treat patients of SCA, and need to be designed to be intuitive to use. However, there are no guidelines or recommendations for the user interface design of these devices, and as such, there are obvious inconsistencies in the aesthetics and functionality, of AEDs currently on the market. This study was designed to assess the variability and determine the visual hierarchy of a range of public access AEDs. This was done by analysing the user's eye gaze behaviour in 400 AED sense-making sessions (10 AEDs * 40 subjects/users).
\end{abstract}

Automated External Defibrillator, Visual Hierarchy, Eye-Tracking, User Interface Design.

\section{INTRODUCTION}

Sudden cardiac arrest (SCA) occurs when the heart unexpectedly stops beating and represents a major cause of death worldwide. It is treated with cardiopulmonary resuscitation (CPR), and delivery of a defibrillating shock. Survival of SCA is timecritical, decreasing rapidly where there are delays in CPR, defibrillation and emergency services arrival (Larsen et al., 1993). Approximately 30,000 SCAs occur outside of medical facilities in the UK (Perkins and Brace-McDonnell, 2015), and as such, automated external defibrillators (AEDs), aimed to be used by the lay person, are now placed in many public locations. It has been reported that public access AEDs are used by untrained or minimally trained users in $62 \%$ of SCA events (Ringh et al., 2015), and therefore it is vital that they are simple in design and intuitive to use. Given the timedependent nature of these events, it is important that untrained or minimally trained users can quickly determine the operation of the AED, and accurately predict prospective tasks simply by looking at the graphical user interface. Research states that a user should be able to do the following when using a device: determine the goal, form the intention to perform specific tasks to achieve this goal, specify the required sequence of these tasks, and execute the planned sequence (Norman, 1986).

There is currently no guidance for the design of AEDs, and there is a wide range on the market, varying in both aesthetics and functionality. Recently the topic of design ethics has been introduced. Mulvenna et al. proposed an "ethical by design manifesto", which aims to ensure the needs of all potential users are considered during technology and product design (Mulvenna, Boger and Bond, 2017). Utilising the principals outlined in this manifesto during medical device design could represent an important step in optimising the production of user-friendly systems. In addition, The Design Council (2015) have stated that the design industry "has a duty to highlight damaging practice wherever it is found, and to continue to educate the public about misleading or harmful aspects of digital products" (Design Council UK, 2015). In the context of AED design ethics, a requirement should be made to ensure that untrained or minimally trained users can navigate through the procedure of providing treatment with an AED in the shortest possible time to avoid delay in life saving therapy.

Eye-tracking technology is commonly used in human-computer interaction $(\mathrm{HCl})$ research to 
measure visual engagement. Eye-tracking has been used in the fields of medical research and healthcare for training, simulation and assessment of clinical decision-making processes (Bond et al., 2014; Currie et al., 2017; McLaughlin et al., 2017), but there has been little research conducted on the use of eye-tracking on medical device design. Using eye-tracking analysis to investigate the user interfaces of medical devices will enable objective quantitative measurements of the variability of the AED user interfaces, as well as insight into their complexity. In particular, the visual hierarchy of AED user interfaces can be determined from eye-gaze metrics. Visual hierarchy is the order in which the information provided on a user interface is processed by the user. The idea of visual hierarchy of user interfaces is based on Gestalt psychological theory. Gestalt proposes that the human brain organises and structures visual elements into shapes or forms (Jackson, 2008). Visual hierarchy may be influenced by design, and as such, user interfaces are typically designed to take a user through a specific task path. We hypothesise that utilising eye-tracking technology to assess the AEDs currently marketed will provide insight to users' cognition, which may lead us to determine recommendations, or best practice guidelines, for AED user interface design.

The aim of this study was to measure visual attention of a public access AED, and determine the visual hierarchy of a range of AEDs currently marketed.

\section{METHODOLOGY}

This study was designed to measure the visual attention and the variability of the AED user interfaces, from which the visual hierarchy was determined. The study underwent ethical review by the Faculty of Computing and Engineering at Ulster University. Forty participants $(n=40)$ from varying demographical and educational backgrounds were recruited.

\subsection{Equipment}

The following equipment was used: Tobii X60 Eyetracker (Tobii AB, Stockholm, Sweden) and the associated Tobii Studio analysis software; laptop with microphone; high-quality images of ten AEDs; and a High Definition monitor.

The Tobii $\mathrm{X} 60$ is a non-intrusive eye-tracker which operates by directing infrared (IR) light into the eye. The light enters the retina, and is reflected, making the pupil appear brighter. The IR light also reflects off the cornea, and after using a vector to determine the distance between the centre of the pupil and the corneal reflection, trigonometric calculations compute the eye-gaze fixation. A number of metrics, such as fixations, saccades and fixation duration can be exported from Tobii Studio. Fixations indicate the locations of visual attention (Duchowski, 2003).

\subsection{Automated External Defibrillators}

The following ten AEDs were selected: HeartSine SAM 500P, Zoll AED Plus, Defibtech Lifeline, IPad SP1, Cardiaid AED, Philips FRx, Cardiac Science Powerheart G5, Mediana A15, Physio Control CR2 and Progetti Rescue SAM AED. All selected AEDs were available for sale in Europe at the time of data collection.

The images were taken from the manufacturers or distributors websites, with the exception of the Physio Control CR2 and the Cardiac Science AED. An image of the Physio Control device was not available at the time of testing, and there were no clear images of the Cardiac Science AED user interface. In these cases, a high-resolution photograph was taken of the device. The AEDs are shown in Figure 3.

\subsection{Determining the Ideal Visual Hierarchy}

The most important features, or Areas of Interest (Aol), on the user interfaces were defined based on the critical tasks associated with AED use. A critical task is defined by the US Food and Drug Administration as a task which if performed incorrectly, or not at all, would or could cause serious harm to the patient or user (U.S. Department of Health and Human Services, 2016). In the case of an AED, the critical tasks carried out by the user are the ability to locate and press the power button, correctly place the pads/electrodes based on the pad placement visuals, enable shock delivery (this may occur either automatically, or by pressing a button), and perform CPR. If any of these tasks are performed incorrectly or not at all, patient survival is seriously jeopardised. Therefore, the most critical Aol in order of importance, are as follows:

1. Power Button

2. Pad Placement Instruction

3. Shock Button

4. CPR Instruction

Although these Aol are the most important for AED usage, all other features of the user interface were captured prior to data recording to allow for more complete determination of a visual hierarchy. An example of the defined Aol is shown in Figure 1. Table 1 shows the Aol of each AED. A total of fifteen different Aol were found across the various AEDs.

\subsection{Pre-Testing Phase}

The study took place in Ulster University in May 2017. Participants were randomly recruited and 
provided consent. The participants were assigned a unique identification number and completed a questionnaire stating demographical information. The Tobii X60 was calibrated for each person prior to beginning data collection. The participant was asked to "make sense" of each image shown on the monitor.

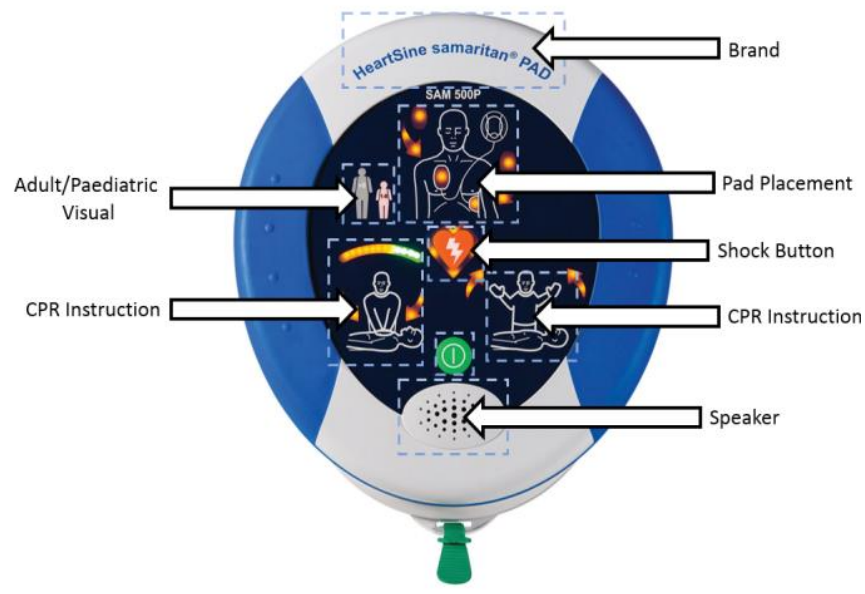

Figure 1: Areas of Interest on the HeartSine SAM 500P predefined using Tobii Studio software.

\subsection{Testing Phase}

The testing occurred in a private room with no interruptions. Each participant was audio recorded for the duration of the test. Images of the ten AEDs were shown one by one on the monitor. Each image was shown for 30 seconds during which the participant had been asked to "make sense" of the user interface. The images were counterbalanced for each participant to avoid order bias. The participant's eye-movements were recorded by the Tobii X60.

\section{RESULTS}

\subsection{Participant Demographics}

Forty participants, $52.5 \%$ male, were recruited for this study. All but one participant reported English as their native language. Participant ages ranged from 18 to 53 years (mean (SD) = 31 (10) years), with one participant unwilling to provide this information. Twenty-nine participants (72.5\%) reported having previously attending CPR training, and twenty-five $(62.5 \%)$ reported having previously attending defibrillation training.

Table 1: Table showing the Areas of Interest for each AED

\begin{tabular}{|c|c|c|c|c|c|c|c|c|c|c|}
\hline $\begin{array}{c}\text { Area of Interest / User Interface } \\
\text { Feature }\end{array}$ & 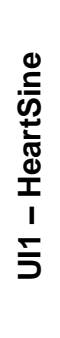 & 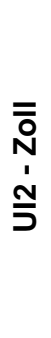 & 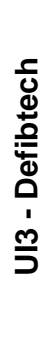 & 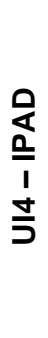 & 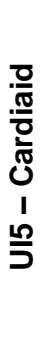 & 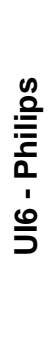 & 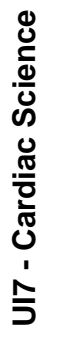 & 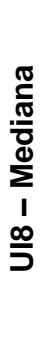 & 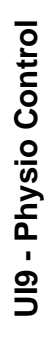 & 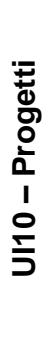 \\
\hline Power Button & $X$ & $X$ & $X$ & $X$ & & $X$ & & & $x$ & $X$ \\
\hline Shock Button & $X$ & $X$ & $X$ & $X$ & $X$ & $X$ & $X$ & $X$ & $X$ & $X$ \\
\hline Rescue Instructions & & $X$ & & & $X$ & & & & & \\
\hline CPR Instructions & $X$ & $X$ & & $X$ & $X$ & & & $X$ & & $x$ \\
\hline Pad Placement Visual Instructions & $x$ & $X$ & & $X$ & $X$ & $X$ & & $X$ & & $X$ \\
\hline Adult/Paediatric Patient & $X$ & $X$ & & $X$ & & & & $X$ & $X$ & \\
\hline Speaker & $X$ & & $X$ & $X$ & $X$ & & $X$ & & $X$ & $X$ \\
\hline Brand & $X$ & & $X$ & $X$ & $X$ & $X$ & & & & $X$ \\
\hline Status Indicator & $X$ & $X$ & & & & & $X$ & $X$ & & \\
\hline Screen & & $X$ & & & & & & & & \\
\hline General Instructions & & & $X$ & $X$ & & $X$ & & & $x$ & $X$ \\
\hline Numbering System & & & & $X$ & & $X$ & & & & $X$ \\
\hline Battery Status Indicator & & & & & & & $X$ & $X$ & & \\
\hline Service Required Status Indicator & & & & & & & $X$ & & & \\
\hline Pad Status Indicator & & & & & & & $X$ & & & \\
\hline
\end{tabular}




\subsection{Eye Gaze Results}

The seven most common Aol were included for analysis. These were the power button, shock button, pad placement instruction, CPR instruction, branding, speaker and the adult/paediatric visual. Four AEDs had the four critical Aol on their user interface: UI1 HeartSine, UI2 Zoll, UI4 IPAD, and Ul10 Progetti. The time-to-first-fixation for each of the four most important Aol on each of these AEDs were summed for each participant, and this is presented in Figure 2.

The mean time-to-first-fixation on each of the Aol was extracted from Tobii Studio and is presented in Table 2. From this, the Visual Hierarchy was determined by listing the mean time-to-firstfixations in order from lowest to highest time. The visual hierarchy for each AED is shown in Figure 3.

The time-to-first-fixation for the seven devices with a power button on the GUI ranged from 1.64 to 11.39 seconds. The Defibtech, Philips and IPad devices have a numbering system on the user interface, where a " 1 " is located beside the power button. Similarly, this numbering system also led to the quickest time-to-first-fixation on the pad placement visual information on the IPAD and Philips devices. Time-to-first-fixation on the pad placement information was longest for the Zoll AED Plus and Mediana A15. In both devices, the pad placement information is not particularly clear. The Zoll AED has eight separate, small images to fixate on, and whilst there is a directed order in which to look at the images as indicated by the arrow, only $20 \%$ of participants followed the intended path to completion.

\section{DISCUSSION}

To the best of our knowledge, this is the first study using eye-tracking technology to assess user interface design of medical devices. Results of the tabulated Aol, the summed Aol time-to-first-fixation data, and the eye-tracking data show that there is a huge variation in AED graphical user interfaces.

As discussed previously, response to SCA is time critical, as chances of survival decrease with every minute that CPR and defibrillation is withheld (Larsen et al., 1993). Therefore, it is vital that an AED can be deployed and used rapidly and without error. We determined that the most important aspect of the AED user interface is the power button. When the user presses the power button, they will hear audio instructions, which will advise the user to their next step. Therefore, if the user does not turn the device on before interacting with the other aspects of the device, such as electrode pads, they will miss vital audio information which could lead to more successful usage. Seven of the ten AEDs (HeartSine, Zoll, Defibtech, IPad, Philips, Physio Control and Rescue SAM) had a power button on the user interface. The remaining three, the Cardiaid, Cardiac Science and Mediana, power-on when the lid is lifted.

Table 2: Table showing the mean Time-to-First-Fixation on each Area of Interest. N/A refers to this AOI not appearing on the AED.

\begin{tabular}{|c|c|c|c|c|c|c|c|}
\hline \multirow[b]{2}{*}{$\begin{array}{c}\text { User } \\
\text { Interface }\end{array}$} & \multicolumn{7}{|c|}{$[x]=$ Visual Hierarchy, with Mean (SD) Time to First Fixation (seconds) } \\
\hline & $\begin{array}{l}\text { Power } \\
\text { Button }\end{array}$ & $\begin{array}{l}\text { Shock } \\
\text { Button }\end{array}$ & $\begin{array}{c}\text { Pad } \\
\text { Placement }\end{array}$ & $\begin{array}{c}C P R \\
\text { Instruction }\end{array}$ & Brand & Speaker & $\begin{array}{c}\text { Adult/ } \\
\text { Paediatric } \\
\text { Visual }\end{array}$ \\
\hline Ul1 HeartSine & $\begin{array}{c}{[6]} \\
10.08(7.58) \\
\end{array}$ & $\begin{array}{c}{[3]} \\
2.998(3.88) \\
\end{array}$ & $\begin{array}{c}{[1]} \\
1.33(1.90) \\
\end{array}$ & $\begin{array}{c}2] \\
2.20(3.24) \\
\end{array}$ & $\begin{array}{c}{[7]} \\
10.49(11.34)\end{array}$ & $\begin{array}{c}4] \\
8.27^{(6.69)}\end{array}$ & $\begin{array}{c}{[5]} \\
9.96(7.57)\end{array}$ \\
\hline UI2 Zoll & $\begin{array}{c}{[4]} \\
11.39(9.52) \\
\end{array}$ & $\begin{array}{c}{[2]} \\
6.19(6.70) \\
\end{array}$ & $\begin{array}{c}{[3]} \\
8.71(6.04) \\
\end{array}$ & $\begin{array}{c}{[1]} \\
3.83(4.58) \\
\end{array}$ & $N / A$ & $N / A$ & $\begin{array}{c}{[5]} \\
16.22(8.13)\end{array}$ \\
\hline UI3 Defibtech & $\begin{array}{c}{[1]} \\
1.98(3.16)\end{array}$ & $\begin{array}{c}{[2]} \\
1.98(3.16)\end{array}$ & $N / A$ & $N / A$ & $\begin{array}{c}{[3]} \\
1.92(2.56)\end{array}$ & $\begin{array}{c}{[4]} \\
13.99(7.84)\end{array}$ & $N / A$ \\
\hline UI4 IPAD & $\begin{array}{c}{[2]} \\
2.49(2.46) \\
\end{array}$ & $\begin{array}{c}{[5]} \\
7.65(5.62) \\
\end{array}$ & $\begin{array}{c}{[1]} \\
0.33(0.84) \\
\end{array}$ & $\begin{array}{c}{[4]} \\
7.63(5.38) \\
\end{array}$ & $\begin{array}{c}{[3]} \\
3.73(5.30) \\
\end{array}$ & $\begin{array}{c}{[7]} \\
10.92(7.25)\end{array}$ & $\begin{array}{c}{[6]} \\
8.51(8.57)\end{array}$ \\
\hline UI5 Cardiaid & $N / A$ & $\begin{array}{c}{[4]} \\
6.72(6.97) \\
\end{array}$ & $\begin{array}{c}{[1]} \\
1.90^{(2.44)} \\
\end{array}$ & $\begin{array}{c}{[2]} \\
2.96(4.08) \\
\end{array}$ & $\begin{array}{c}{[5]} \\
8.46(11.14) \\
\end{array}$ & $\begin{array}{c}{[3]} \\
6.62(7.51)\end{array}$ & $N / A$ \\
\hline UI6 Philips & $\begin{array}{c}{[2]} \\
1.64(1.41)\end{array}$ & $\begin{array}{c}{[3]} \\
7.55(5.82)\end{array}$ & $\begin{array}{c}{[1]} \\
0.76(1.45)\end{array}$ & $N / A$ & $\begin{array}{c}{[4]} \\
14.21(11.43)\end{array}$ & $N / A$ & $N / A$ \\
\hline $\begin{array}{l}\text { Ul7 Cardiac } \\
\text { Science }\end{array}$ & $N / A$ & $\begin{array}{c}{[1]} \\
2.62(4.21) \\
\end{array}$ & $N / A$ & $N / A$ & $\begin{array}{c}{[3]} \\
5.10^{(5.32)}\end{array}$ & $\begin{array}{c}{[2]} \\
4.97(5.72)\end{array}$ & $N / A$ \\
\hline UI8 Mediana & $N / A$ & $\begin{array}{c}{[2]} \\
7.724(6.63)\end{array}$ & $\begin{array}{c}3] \\
8.73(6.90)\end{array}$ & $\begin{array}{c}{[4]} \\
12.63(7.37)\end{array}$ & $N / A$ & $N / A$ & $\begin{array}{c}11] \\
4.33(4.72)\end{array}$ \\
\hline $\begin{array}{l}\text { UI9 Physio } \\
\text { Control }\end{array}$ & $\begin{array}{c}{[2]} \\
10.97(9.34) \\
\end{array}$ & $\begin{array}{c}{[1]} \\
9.60^{(7.87)}\end{array}$ & $N / A$ & $N / A$ & $N / A$ & $\begin{array}{c}{[3]} \\
14.73(10.12)\end{array}$ & $\begin{array}{c}{[4]} \\
15.37(8.81) \\
\end{array}$ \\
\hline Ul10 Progetti & $\begin{array}{c}{[5]} \\
11.27(6.45)\end{array}$ & $\begin{array}{c}{[3]} \\
5.21^{(5.39)}\end{array}$ & $\begin{array}{c}{[1]} \\
1.38(1.90)\end{array}$ & $\begin{array}{c}{[2]} \\
4.95(4.44)\end{array}$ & $\begin{array}{c}{[6]} \\
19.31(14.13)\end{array}$ & $\begin{array}{c}{[4]} \\
5.54(5.78)\end{array}$ & $N / A$ \\
\hline
\end{tabular}




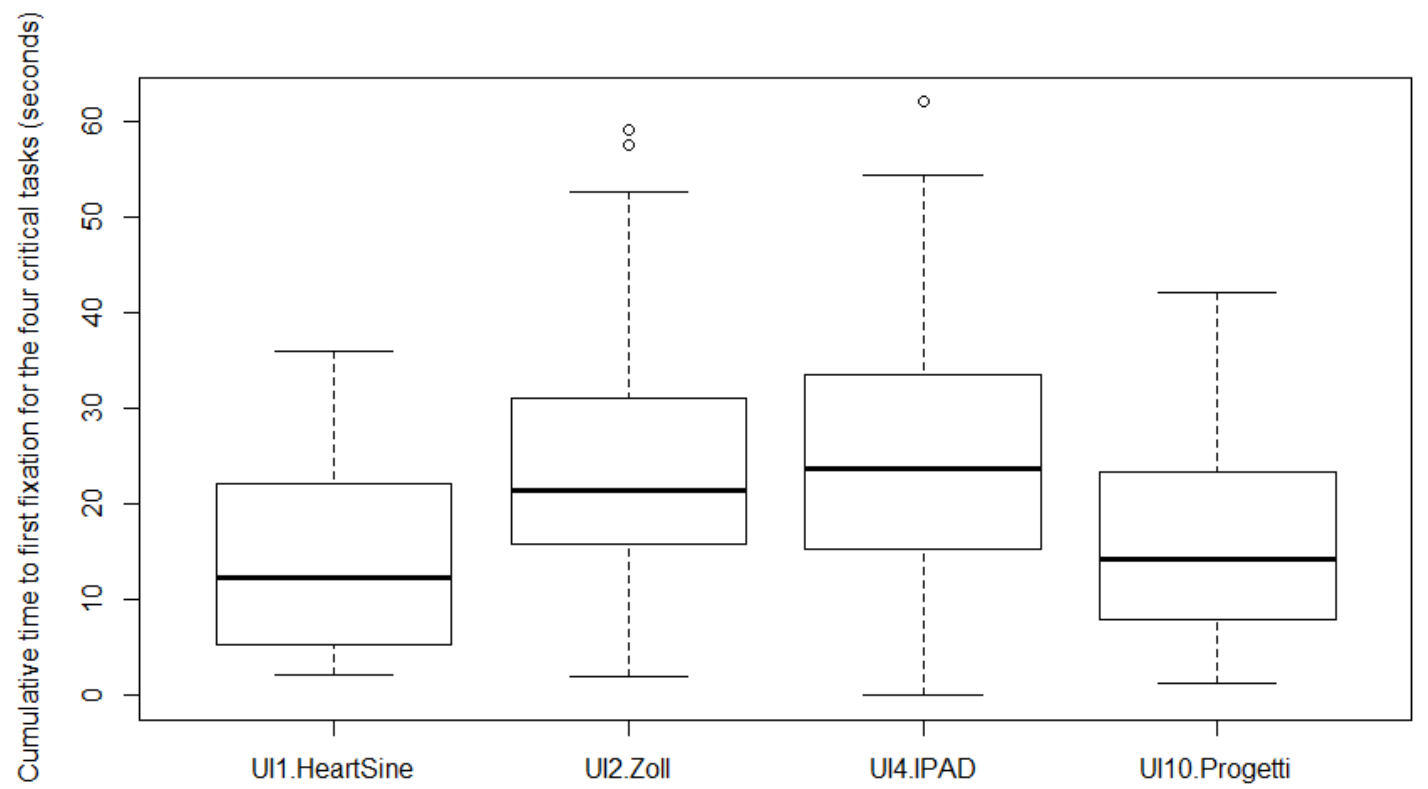

Figure 2: Cumulative time to first fixation on the four critical Areas of Interest.

The Physio Control AED has a power button, but also powers on when the lid is lifted.

The ethics relating to the lack of guidance and design auditing of AED user interfaces should be questioned. Approximately 30,000 SCAs occur outside a medical facility in the UK (Perkins and Brace-McDonnell, 2015). The proportion of UK public who have received training in CPR defibrillation training has been reported to be approximately 22\% (Hawkes et al., 2017). Therefore, in many cases, SCAs are witnessed and responded to by untrained lay rescuers. If these rescuers have previously attended CPRdefibrillation training, they will have mostly likely only received training on one specific AED. However, in a true SCA event, the rescuer is expected to perform quickly and effectively on whichever AED is readily available. Previous usability study data has demonstrated the negative effect of differing design on successful defibrillation. A simulation study was conducted in which participants interacted with four different AEDs. The proportion of successful shock delivery ranged from $44 \%$ to $100 \%$, with median time-to-first-shock ranging from 93 to 210 seconds. Of note, the AED with electrode pads that were very different in design from the other AEDs had the lowest proportion of successful shock and the longest median time-to-first-shock (Andre et al., 2004). Ensuring some standardisation in design of AED user interfaces, and accessories such as electrode pads could lead to better performance by nonmedically trained rescuers, and ultimately improve survival outcomes from SCA.
Although there are some international standards related to design of medical devices, these focus particularly on risk management and control methods relating to the design process (BSI: British Standards, 2012). To the best of our knowledge, there are no specific design methodology guidelines related to medical devices, which should lead to the development of superior products. Developers of consumer devices such as mobile phones and laptops, including Apple and Microsoft, have outlined their own best practice design guidelines for developers (Microsoft, 2013; Apple, 2017), and have proven success with user adoption and performance with their products. Adoption of such methodologies in the medical device industry may lead to better usability, more successful device usage, and in the case of the AED, improved survival outcomes.

\section{Limitations}

A minor limitation of this study is the range of images used. The images were selected from the manufacturer's website with the exception of the Physio Control and Cardiac Science AEDs and are of varying quality. This also meant that some AEDs are displayed in a manner in which they are not usually available. For example, the HeartSine SAM 500P will never have all LEDs lit, and the lid is not shown in the Cardiac Science AED. In addition, all images were scaled to $1400 \times 900$ pixels to ensure they fit the HD monitor screen.

This study also did not assess the effect of animated user interfaces, lights, or sound on the visual hierarchy of these AEDs. 


\section{Future Work}

Further recruitment of participants who are unfamiliar with AEDs and have had no previous CPR or defibrillation training, would enable comparison of the eye-gaze metrics of those participants who were familiar with AEDs and had received previous training.

Due to the limitations relating to the choice of images used for this study, a future study may be conducted using images presented at $100 \%$ scale of each individual AEDs physical size to determine if the scaled images affected the visual hierarchy testing. In addition, use of a wearable eye-tracker and the actual device rather than images on a screen may also allow for deeper analysis of the user interfaces.

Finally, proposed designs of an AED user interface with optimised visual hierarchy could be used to repeat the study to determine if an "ideal" user interface exists.

\section{CONCLUSION}

This study is, to the best of our knowledge, the first to have used eye-tracking technology to assess the user interface of public access defibrillation devices. These results highlight the variation in the functionality and design of public access defibrillators, which may have ethical implications.

\section{REFERENCES}

Andre, A. D. et al. (2004) 'Automated external defibrillator use by untrained bystanders: Can the public-use model work?', Prehospital Emergency Care, 8(3), pp. 284-291. doi: 10.1016/j.prehos.2004.02.004.

Apple (2017) UI Design Do's and Don'ts - Apple Developer. Available at: https://developer.apple.com/design/tips/ (Accessed: 7 March 2018).

Bond, R. R. et al. (2014) 'Assessing computerized eye tracking technology for gaining insight into expert interpretation of the 12-lead electrocardiogram: An objective quantitative approach', Journal of Electrocardiology. Elsevier Inc., 47(6), pp. 895-906. doi: 10.1016/j.jelectrocard.2014.07.011.

BSI: British Standards (2012) 'BS EN ISO 14971:2012: Application of Risk Management to Medical Devices'.

Currie, J. et al. (2017) 'Eye Tracking the Visual Attention of Nurses Interpreting Simulated Vital
Signs Scenarios: Mining Metrics to Discriminate Between Performance Level', IEEE Transactions on Human-Machine Systems, pp. 1-12. doi: 10.1109/THMS.2017.2754880.

Design Council UK (2015) The ethics of digital design, Design Council UK. Available at: https://www.designcouncil.org.uk/news-

opinion/ethics-digital-design (Accessed: 27 March 2018).

Duchowski, A. T. (2003) Eye Tracking Methodology: Theory and Practice. Springer. doi: 10.1007/978-3-319-57883-5.

Hawkes, C. et al. (2017) 'Attitudes to CPR and public access defibrillation: A survey of the UK public', Resuscitation. Elsevier Ireland Ltd, 118, p. e39. doi: 10.1016/j.resuscitation.2017.08.101.

Jackson, I. (2008) 'Gestalt-A Learning Theory for Graphic Design Education', International Journal of Art \& Design Education, 27(1), pp. 63-69. doi: 10.1111/j.1476-8070.2008.00558.x.

Larsen, M. P. et al. (1993) 'Predicting survival from out-of-hospital cardiac arrest: A graphic model', Annals of Emergency Medicine, 22(11), pp. 16521658. doi: 10.1016/S0196-0644(05)81302-2.

McLaughlin, L. et al. (2017) 'Computing eye gaze metrics for the automatic assessment of radiographer performance during X-ray image interpretation', International Journal of Medical Informatics. Elsevier Ireland Ltd, 105, pp. 11-21. doi: 10.1016/j.jmedinf.2017.03.001.

Microsoft (2013) 'Microsoft design principles'. Available at: https://www.microsoft.com/enus/design (Accessed: 7 March 2018).

Mulvenna, M., Boger, J. and Bond, R. (2017) 'Ethical by Design', in Proceedings of the European Conference on Cognitive Ergonomics 2017 - ECCE 2017, pp. 51-54. doi: 10.1145/3121283.3121300.

Norman, D. A. (1986) 'Cognitive engineering', User centered system design, 31, p. 61.

Perkins, G. D. and Brace-McDonnell, S. J. (2015) 'The UK out of Hospital Cardiac Arrest Outcome (OHCAO) project', BMJ Open, 5(10). doi: 10.1136/bmjopen-2015-008736.

Ringh, M. et al. (2015) 'Survival after Public Access Defibrillation in Stockholm, Sweden - A striking success', RESUSCITATION, 91, pp. 1-7. doi: 10.1016/j.resuscitation.2015.02.032.

U.S. Department of Health and Human Services (2016) Applying Human Factors and Usability Engineering to Optimize Medical Device Design, FDA Guidance for Industry. doi: 10.1177/1071181311551334. 

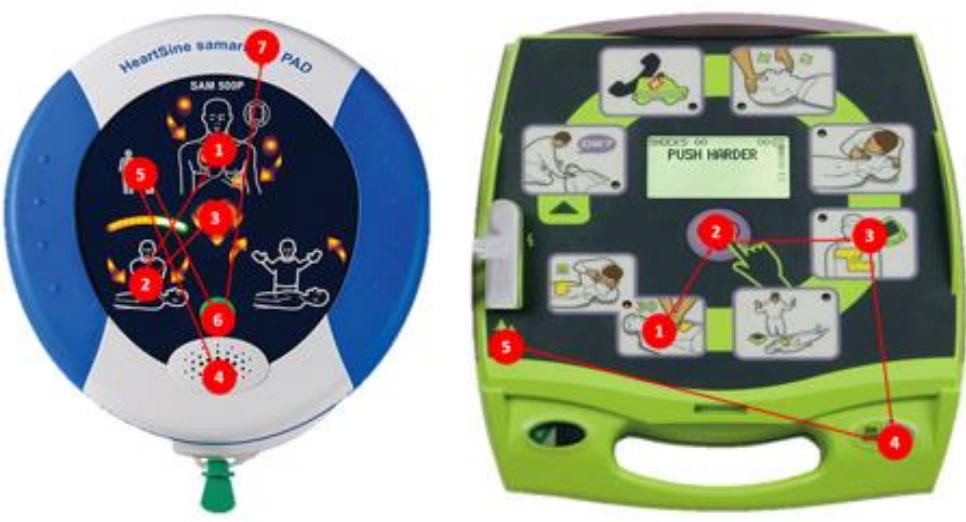

UI 1: HeartSine SAM 500P

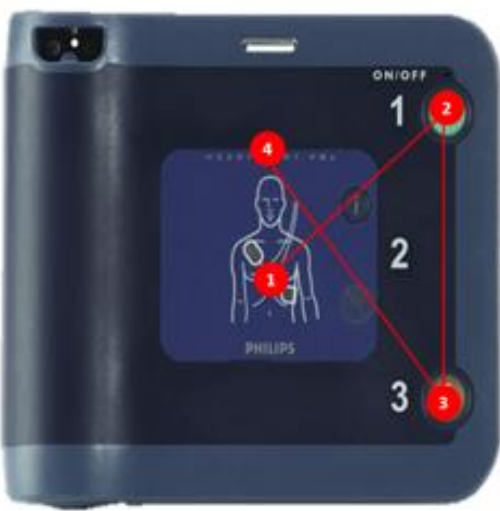

UI 6: Philips FRx

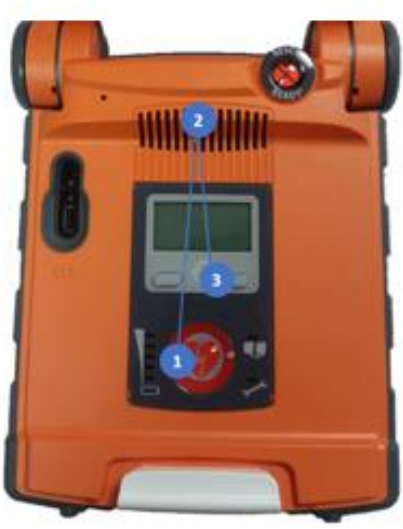

UI 7: Cardiac Science Powerheart G5

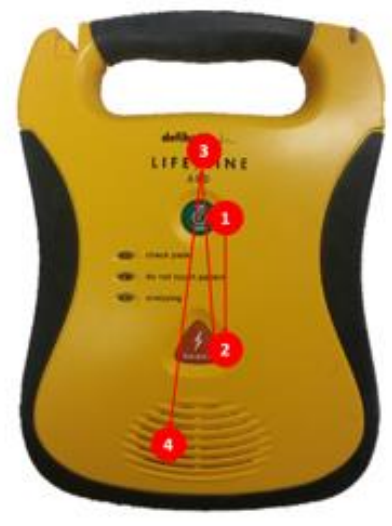

UI 3: Defibtech Lifeline

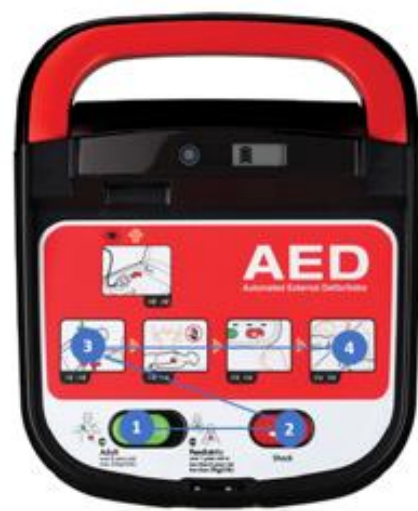

UI 8: Mediana A15

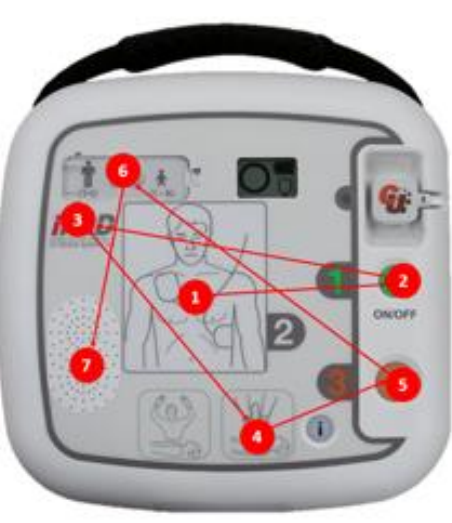

UI 4: IPAD SP1

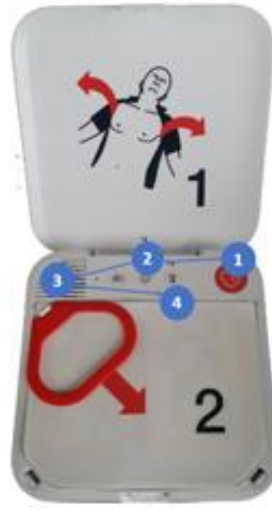

UI 9: Physio Control CR2

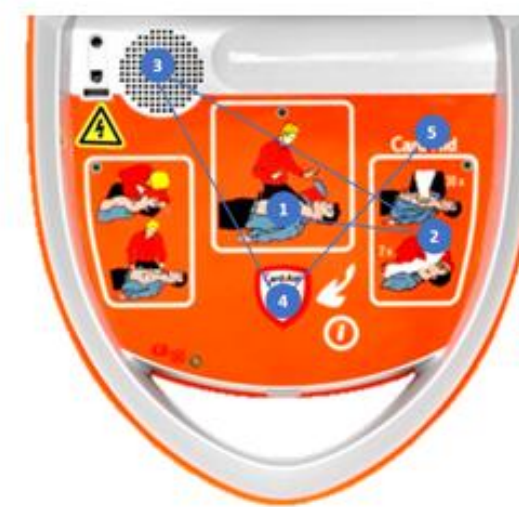

UI 5: Cardiaid AED

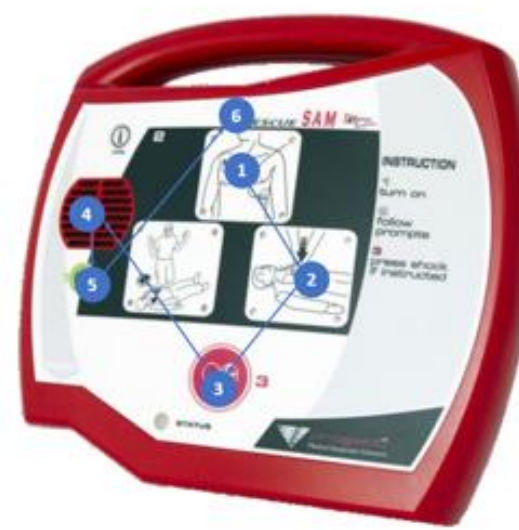

UI 10: Progetti Rescue SAM AED

Figure 3: The visual hierarchy determined for each AED. UI1 HeartSine SAM 500P, UI2 Zoll AED Plus, UI3 Defibtech Lifeline, UI4 IPAD SP1, UI5 Cardiaid AED, UI6 Philips FRx AED, UI7 Cardiac Science Powerheart G5, UI8 Mediana A15 HeartOn AED, UI9 Physio Control, UI10 Progetti Rescue SAM AED 